\title{
Family study of inherited syndrome with multiple congenital deformities: symphalangism, carpal and tarsal fusion, brachydactyly, craniosynostosis, strabismus, hip osteochondritis
}

\author{
V. VENTRUTO, R. DI GIROLAMO, B. FESTA, A. ROMANO, G. SEBASTIO, and \\ L. SEBASTIO \\ From Dipartimento di Ematologia degli Ospedali Riuniti di Napoli, Servizio di Immunopatologia e Genetica Ematologica, \\ Naples; and Dipartmento di Ematologia degli Ospedali Riuniti di Napoli, Divisione di Ematologia, Naples; Servizio di \\ Radiologia degli Ospedali Riuniti di Napoli, Naples, Italy \\ Summary. A syndrome of brachydactyly (absence of some middle or distal \\ phalanges), aplastic or hypoplastic nails, symphalangism (ankylosis of proximal \\ interphalangeal joints), synostosis of some carpal and tarsal bones, craniosynostosis, \\ and dysplastic hip joints is reported in five members of an Italian family. It may \\ represent a previously undescribed autosomal dominant trait.
}

Brachydactyly and symphalangism are wellknown hereditary anomalies of the hands and feet. In brachydactyly, digits or specific phalanges may be affected, sometimes in association with shortening of metacarpal and metatarsal bones, and classification has been based on specific patterns (Bell, 1951). The thumbs and big toes are not usually involved. There may be various degrees of dysplasia of the nails (Cuevas-Sosa and Garcia-Segur, 1971). Symphalangism is the result of failure of differentiation of the interphalangeal joints, and leads to ankylosis between proximal or, more rarely, distal interphalangeal joints. Other congenital anomalies may be associated with symphalangism, the most common of which is synostosis of the carpal and tarsal bones.

We report here five cases in three generations of a syndrome of brachydactyly with symphalangism, and other features including craniosynostosis. This may be a hitherto undescribed autosomal dominant trait.

\section{Family study}

The propositus, III.3, was first seen because of acute leukaemia, and the bone anomalies were observed in four other members of her family in three generations (Fig. 1). The anomalies were in the hip, the cranium, and both hands and feet (Fig. 2-5). They varied in severity, and the malformations were not symmetrical in all affected individuals (Appendix). The nails presented various degrees of dystrophy (including total absence), solely on the brachydactylous fingers, while they appeared normal where there was symphalagism without brachydactyly (IV.4). All five individuals showed craniosynostosis, hip dysplasia, and pes planus, and had pain on walking. The least affected family member is IV.4 who had symphalangism and clinodactyly, but lacked brachydactyly, had normal nails, and showed partial craniosynostosis. Hip dysplasia in II.7 caused a severe joint dislocation. The stature, weight, and intelligence are normal in all subjects. III.1 and his daughter IV.1 showed strabismus, but there was no defect of vision or hearing.

Laboratory data were normal including serum calcium, serum phosphorus, and serum phosphatase. The

I

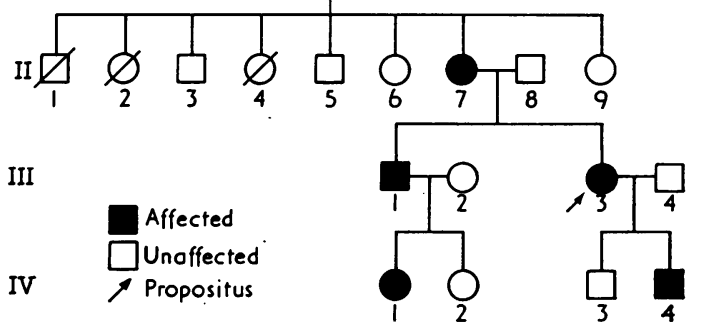

Fig. 1.

Received 11 December 1975. 

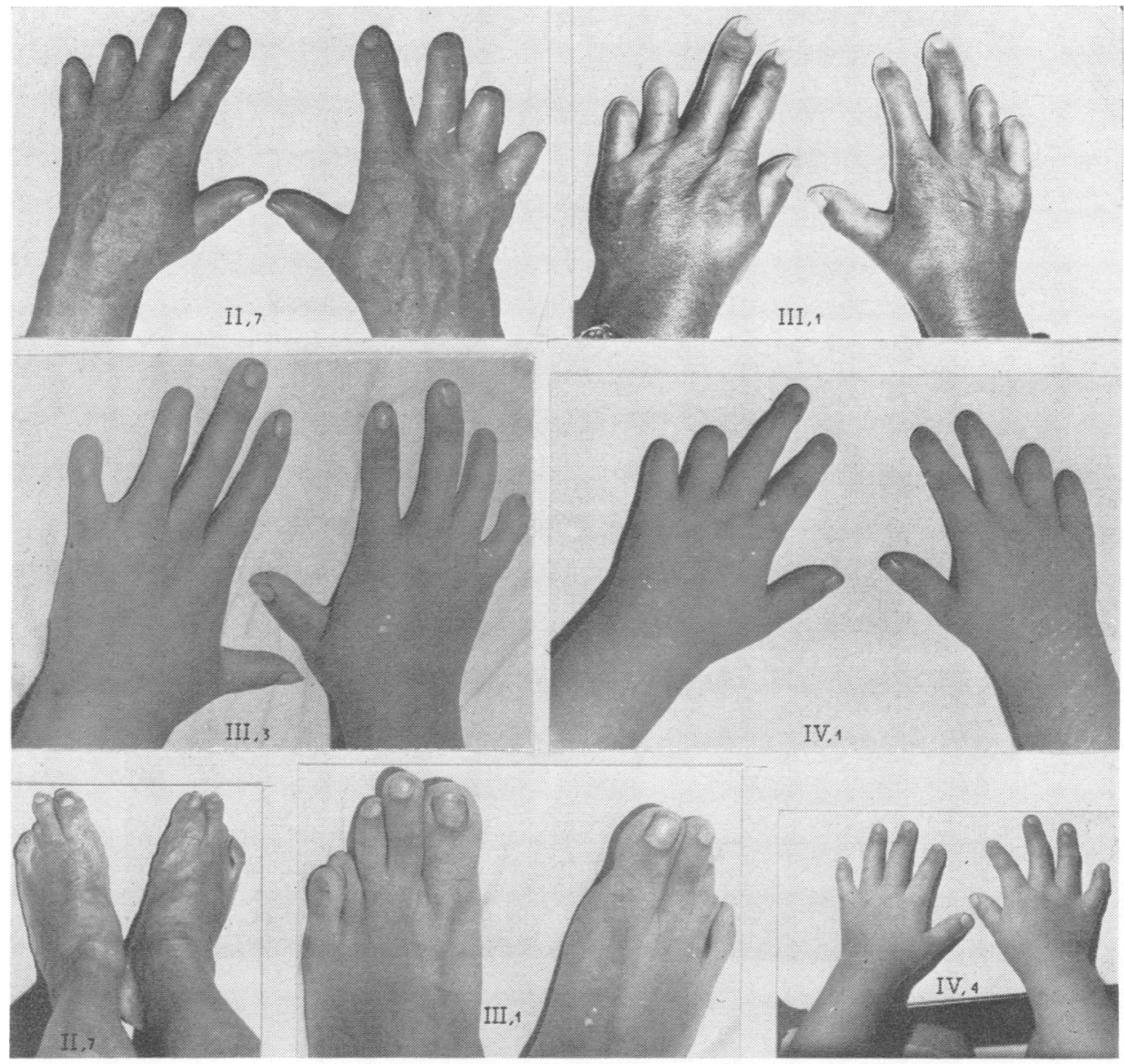

FIG. 2.

electroencephalogram was also normal, as were the pattern of the sensitive organs. A normal karyotype was seen on two affected individuals (II.7 and III.1) by banding techniques. The following genetic markers were examined: adenylate kinase, malate dehydrogenase, phosphohexose isomerase, phosphoglucomutase, adenosine deaminase, phosphoenolpyruvate, aspartate transaminase, alanine transaminase, diaphorase. Unfortunately, no information regarding linkage was obtained from these data.

Dermatoglyphic patterns showed some unusual features, including: absence of some digital triradii and an increase in the axial triradius angle; a type 'epsilon' configuration by D-line exit in III.3; characteristic patterns on the brachydactylic fingers; the absence of flexion creases at interphalangeal joints; the presence of triradius in some of these areas; and the high incidence of whorls. There were no patterns in the thenar or hypothenar areas. 


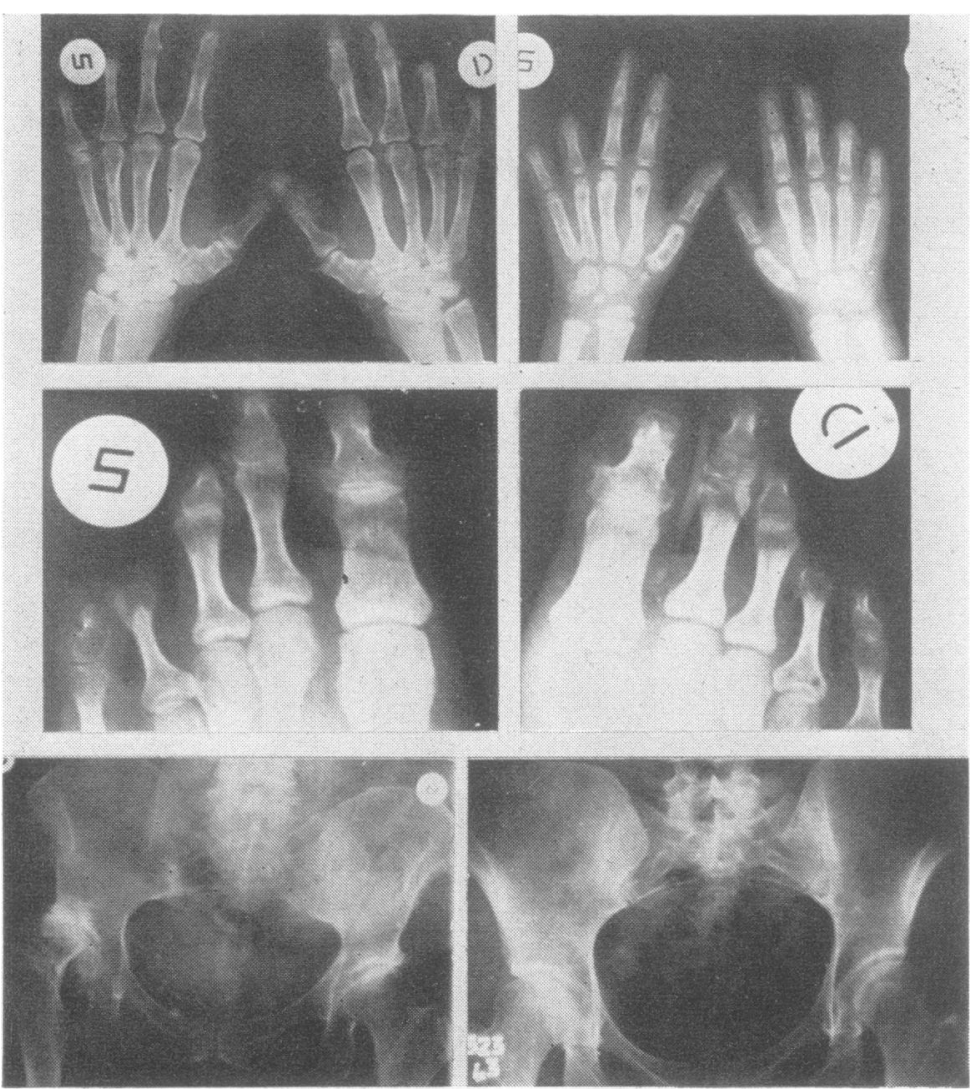

FIG. 3.

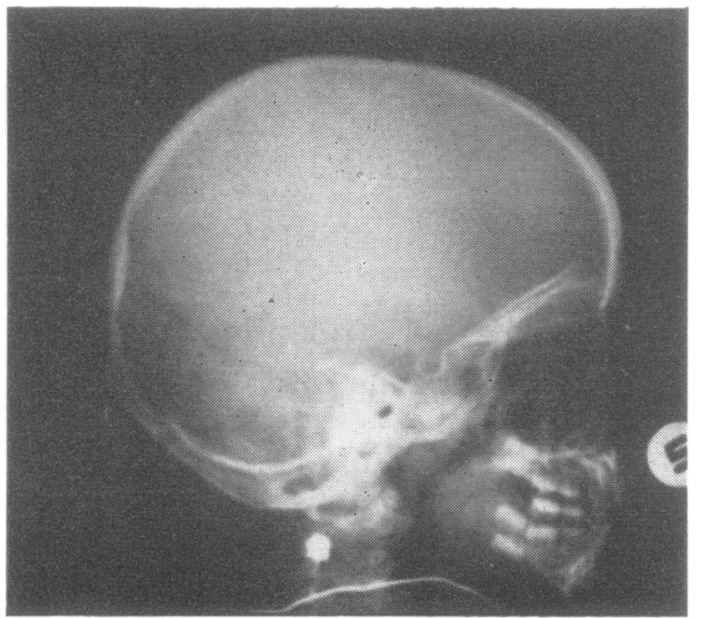

FIG. 4. 


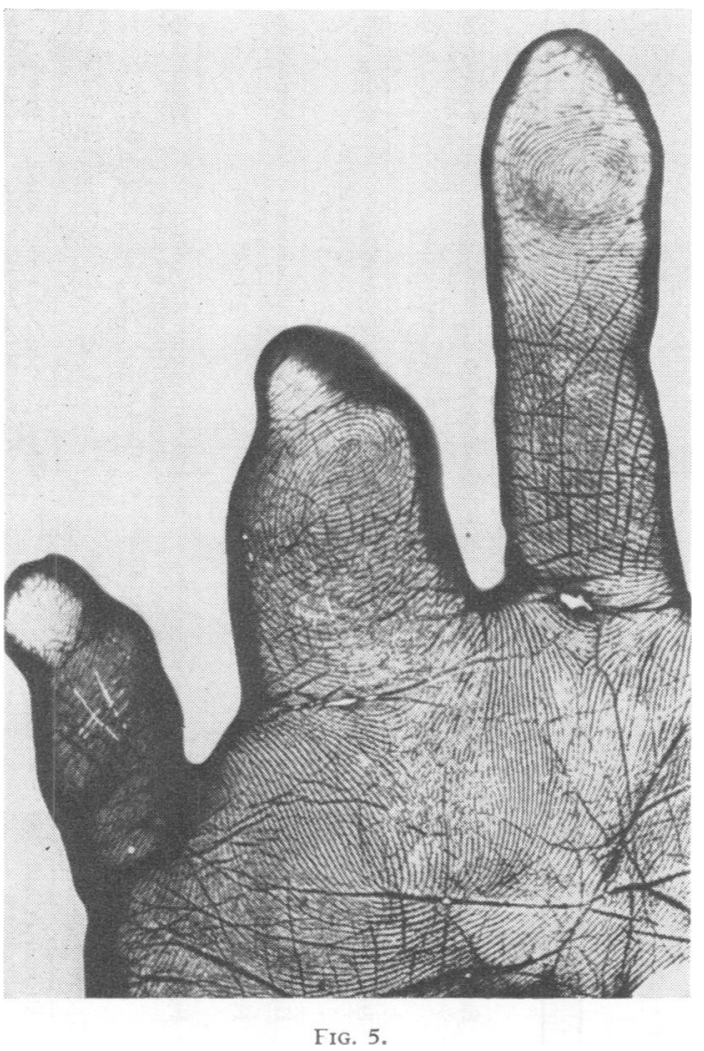

type reported previously. The disorder is located both in hands and feet, though the thumbs and great toes are unaffected. To our knowledge, strabismus and craniosynostosis have not been described before in connexion with symphalangism and/or brachydactyly. As for the dysplasia of hip joints, it is noteworthy that the femoral heads appeared to be normal; the anomaly involved the acetabular structure (osteochondritis). The hip abnormality is, therefore, distinct from that described by Robinson et al, in which the changes were similar to those of Perthes' disease (osteochondritis of the capital femoral epiphysis).

The distribution in the pedigree is consistent with an autosomal dominant trait, with some variation in gene expressivity. It may have arisen as a new mutation in generation $I$.

The authors thank Dr Silvana Santachiara, Laboratorio di Genetica Biochimica ed Evoluzionistica di Pavia, for her help in the study of genetic markers, and $\mathrm{Dr} \mathrm{D}$. M. Bannerman, Division of Medical Genetics, State University of New York at Buffalo, for his advice and criticism of the manuscript.

\section{REFERENCES}

Bell, J. (1951). On brachydactyly and symphalangism. In Treasury of Human Inheritance, Vol. 5, p. 1. Cambridge University Press, London and New York.

Cuevas-Sosa, A. and Garcia-Segur, F. (1971). Brachydactyly with absence of middle phalanges and hypoplastic nails. Fournal of Bone and foint Surgery, 53, 101-105.

Haws, D. V. (1963). Inherited brachydactyly and hypoplasia of the bones of the extremities. Annals of Human Genetics, 26, 201-211.

McKusick, V. A. (1975). Mendelian Inheritance in Man, 4th ed., p. 43. The Johns Hopkins Press, Baltimore.

Robinson, G. C., Wood, B. J., Miller, J. R., and Baillie, J. (1968). Hereditary brachydactyly and hip disease. fournal of Pediatrics, 72, 539-543. 


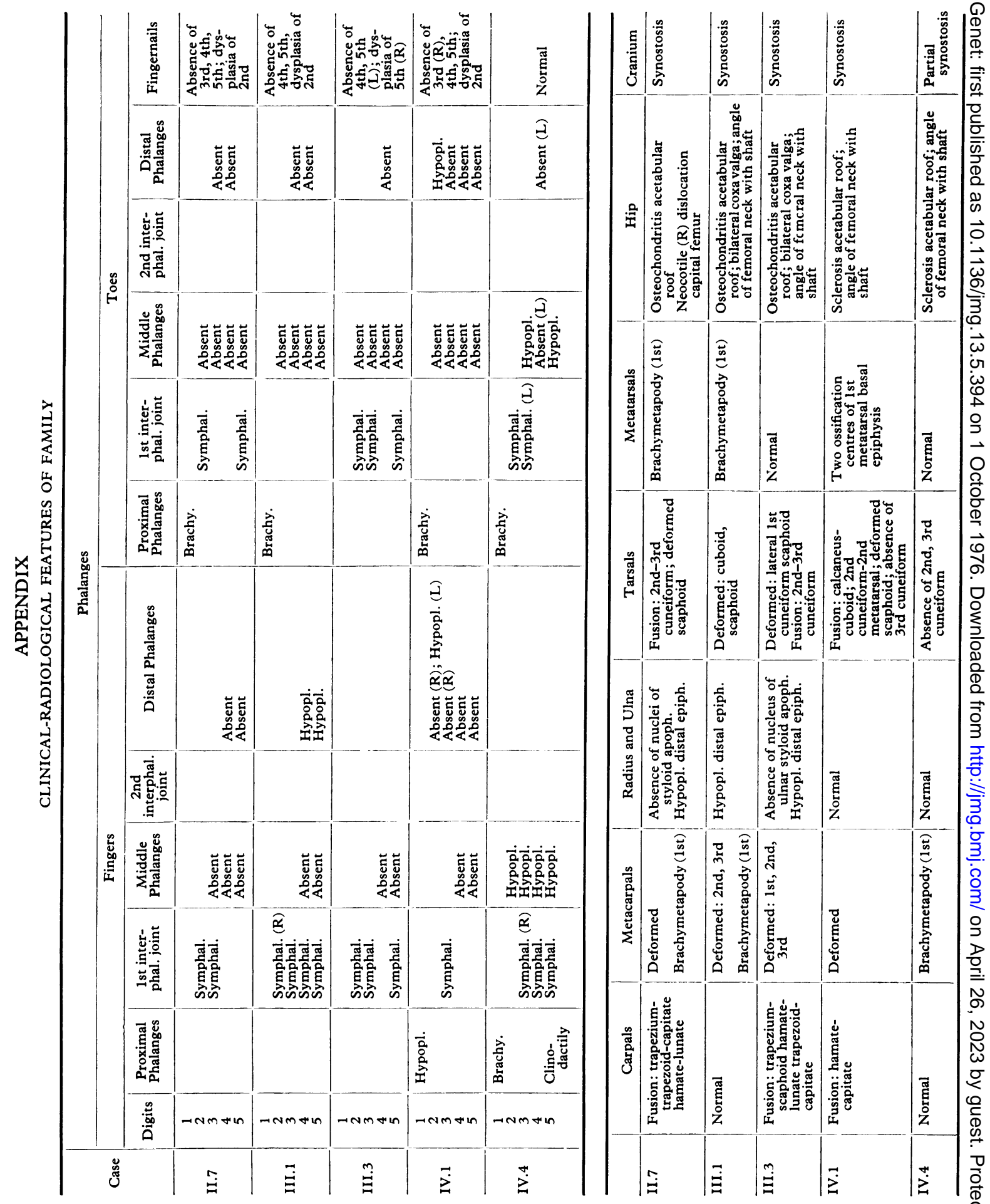

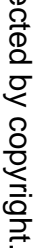

